\title{
Path way for Critical Review on Early Prediction of Bone Density Through Various Techniques
}

\author{
S Ashmad ${ }^{1}$ and Ramesh. G.P2 \\ ${ }^{1}$ Department of Electronics and Communication Engineering, \\ St.Peter's Institute of Higher Education and Research, Chennai, Tamil Nadu, India \\ ${ }^{2}$ Department of Electronics and Communication Engineering, St.Peter's \\ Institute of Higher Education and Research, Chennai, Tamil Nadu, India
}

\section{ABSTRACT}

Recent advancements in medical applications like detecting bone density in early stages is utmost necessary to save human lives. In this connection finding the amount of mineral or calcium content present in the bone or bone tissue plays a critical role, for that Bone Densitometer are utilizes to find exact Bone Mineral Density(BMD) or bone density values that give information about the structure of bone. Generally, Bone Densitometer gives two forms of results one is T-score and the other is Z-score. If the value of T-score and Z-score is less than the threshold value, then the density of bone is reduced and it will lead to chances of bone fracture. Frequently used bone densitometers are like Dual Energy X-ray Absorptiometry (DEXA) and Radiographic machines like CT and MRI. This review article describes the volumetric and critical review on various techniques exists so far to determine the bone density from these it was observed that CT and MRI are most expensive and it generates more radiation which causes problems for human. Till now in literature the recorded accuracy with these methods are low. Finally, the inference drawn from literature had given a thought thatthe usage of normal X-Ray machine when compared to existed methods may give better accuracy.

KEY WORDS: BONE MINERAL DENSITY (BMD), DUAL ENERGY X-RAY ABSORPTIOMETRY (DEXA) , CT SCAN AND MRI.

\section{INTRODUCTION}

BMD indicates bone strength. It measures how much minerals are present in the bone. Minerals like calcium and phosphorus helps to maintain bones strong. Bones which are have less mineral content then it become weaker bones and requires lots of minerals ie., high volume of bone mineral. Weaker bones are one of the major causes of fractures in elderly. BMD test also referred

\section{ARTICLE INFORMATION}

*Corresponding Author: rameshgp@yahoo.com

Received 9th Oct 2020 Accepted after revision 26th Dec 2020

Print ISSN: 0974-6455 Online ISSN: 2321-4007 CODEN: BBRCBA

Thomson Reuters ISI Web of Science Clarivate Analytics USA and Crossref Indexed Journal

\section{Clarivate
Analytics}

NAAS Journal Score 2020 (4.31)

A Society of Science and Nature Publication,

Bhopal India 2020. All rights reserved.

Online Contents Available at: http//www.bbrc.in/

Doi: http://dx.doi.org/10.21786/bbrc/13.13/42 to as bone density test that detects osteoporosis and the word osteoporosis word derived from Greek Word, which means "porous bone."

Determination of BMD helps to detect osteoporosis and to evaluate upcoming fracture risk. Even if the BMD test is not $100 \%$ precise but it will be determine that whether someone will have a fracture in future or not. The bone density test offers two parameters: the T-score and the Z-score. The T-score defines typical bone mass, low bone mass or osteopenia and osteoporosis (Parameshachari et al. 2020; and Prabu et al. 2019). The results compared with bone density of average bone density of healthy young adults of same gender. The Table 1 shows T-scores used to determine the state of the bone.

Typical bone density test used to diagnose the bone fracture when T-score is -1.0 and beyond. If the value of T-score is between -1 to -2.5 indicates low bone mass

$$
283
$$


called osteopenia.The T-score below -2.5 shows that the bone becomes porous called osteoporosis. The Z-score describes and compares the results of bone density test with same gender and age of normal population. If the $\mathrm{Z}$-score is less than two then it gives warning indication that the bone having less porosity.

Table 1. Shows Bone Density Values

\begin{tabular}{|l|c|}
\hline T-score & BMD Indication \\
\hline $\begin{array}{l}\text { Greater than or } \\
\text { Equal to } 1\end{array}$ & Healthy Bone Density \\
\hline $\begin{array}{l}\text { Less than }-1 \text { and } \\
\text { greater than }-2\end{array}$ & $\begin{array}{c}\text { Low bone mass } \\
\text { (Osteapenia) }\end{array}$ \\
\hline $\begin{array}{l}\text { Less than or equal to -3 } \\
\text { Less than }-4\end{array}$ & $\begin{array}{c}\text { Presence of Osteoporosis } \\
\text { Severe or established } \\
\text { Osteoporosis }\end{array}$ \\
\hline
\end{tabular}

Figure 2: Flow process for diagnosing Bone Density

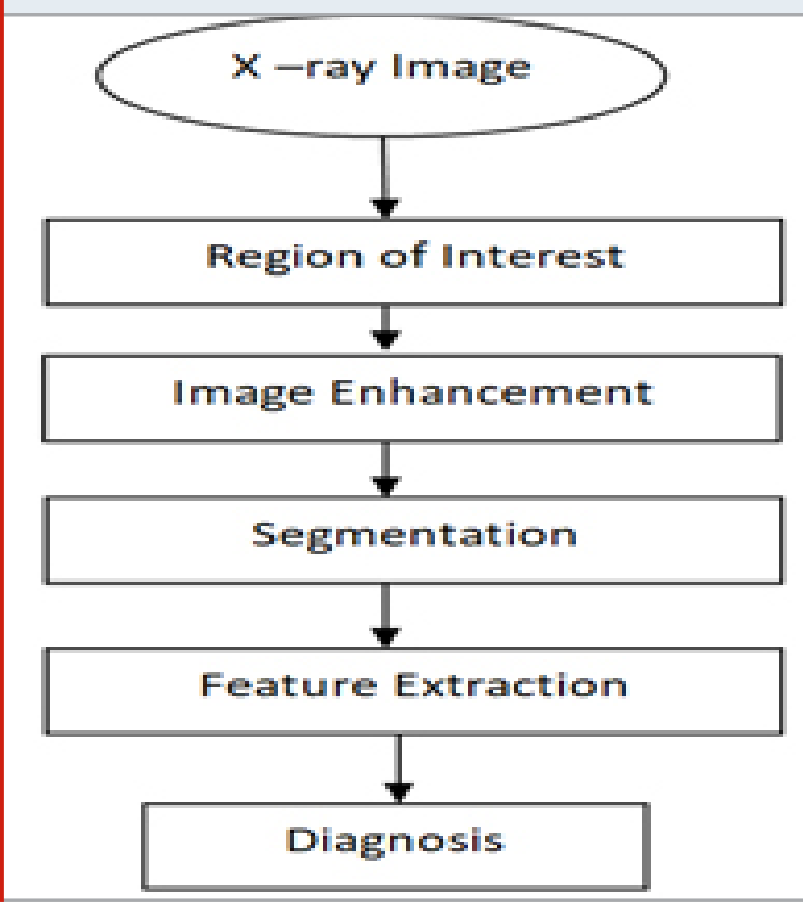

\section{METHODOLOGY}

The input is the X-ray image that is obtained from DEXA or CT and this can be diagnosed with various methods and algorithms. In DEXA scan number of parameters is to be considered like hip bone, spine-lumbar bone, vertebrae or whole body. In general after scanning of bone image using DEXA, an automatic system will be run to calculate BMD values that is T-score and Z-score.

Standard value of T- score value represented in equation (1).

$$
T-\text { score }=\frac{(\text { BMD-young Normal Mean })}{\sigma}
$$

Where,

BMD is Bone Mineral Density and " $\sigma$ " is standard deviation.

BMD is measured using standard equation with respect to Mean Gray Level (MGL). The BMD value can be estimated as shown in equation (2).

$\mathrm{BMD}=0.0035^{*} \mathrm{X}+\mathrm{S}$

Where,

$\mathrm{X}=$ Mean(MGL)

The figure 2 represents the flow of diagnosing the osteoporosis like Region of Interest (ROI), Image enhancement, segmentation, feature extraction or texture analysis and finally a diagnosed image can be obtained. To get accurate and reliable quantitative information, image processing algorithms are applied to remove artifacts and degradation like blur and noise. The ROI comes under pre-processing that can be defined in the $\mathrm{X}$ -ray bone image that considered as main quality of image than other region called background. Some examples of ROI is shown in figure 3.

Figure 3: Examples of Region of Interest(ROI) of different bones

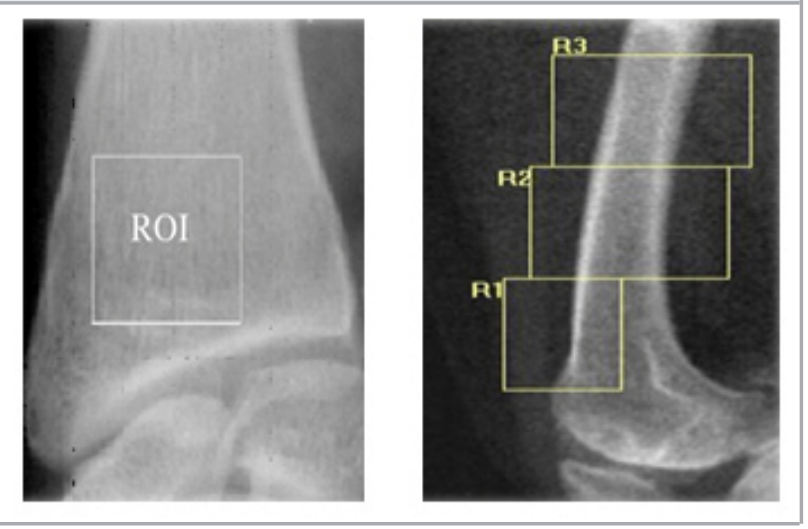

Region of interest(ROI) used to identify the bone that is having low resolution. By using various techniques like manual, semi-automated, or automated, the ROI of bone is segmented.The ROI, fracture, and BMD is estimated and predicted by using various image enhancement techniques which improves the quality of the image. Normal segmentation methods fails to produce accurate results of bone images. Consequently automated image enhancement techniques are used to produce precise results. Consider, 'I' as a MXN size input image and ' $m$ ' is average intensity that is shown in equation (3).

$$
m=\sum_{r=0}^{M-1} \frac{\sum_{C=0}^{N-1} l(r, c)}{M x N}
$$

$H=H_{L} \cup H_{O}$ 
Where

$\mathrm{H}_{\mathrm{L}}=\left\{\mathrm{H}_{0}+\mathrm{H}_{1}+\ldots \ldots \ldots+\mathrm{H}_{\mathrm{m}}\right\}$

$\mathrm{H}_{\mathrm{U}}=\left\{\mathrm{H}_{\mathrm{m}+1}+\mathrm{H}_{\mathrm{m}+2}+\ldots \ldots . .+\mathrm{H}_{\mathrm{I}-1}\right\}$

' $m$ ' refers to mean intensity and used as separation point which divides the histogram ' $\mathrm{H}$ ' in two parts $\mathrm{H}_{\mathrm{L}}$ and $\mathrm{H}_{\mathrm{U}}$ referred to as sub histograms indicated in equations (5) and (6).

Area Overlap and Confusion matrix method indicates the performance of bone density of vertebral. This can be illustrated in equations (7) and (8).

$$
\text { Area Oerlap }=\left|\frac{\mathrm{A} \cap \mathrm{B}}{\mathrm{A}}\right| \times 100
$$

\section{Where}

' $A$ ' is an output image and

' $\mathrm{B}$ ' is an equivalent ground - truth images.

$$
\text { Precision }=\frac{T P}{T P+F P} \times 100
$$

Where,

$$
\begin{aligned}
& \text { TP - True Positive } \\
& \text { FP - False Positive }
\end{aligned}
$$

- True Positive (TP): Properly identified as Osteoporosis.

- False Positive (FP): Properly recognized as nonosteoporosis.

Figure 4 and 5: Full spine X-ray image and Spine ROI Detection

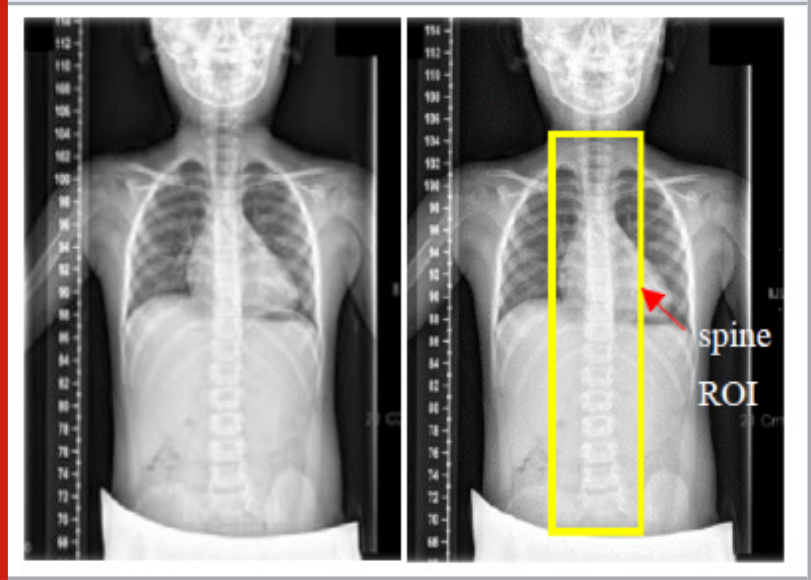

There are various segmentation methods are available like space clustering, region growing, edge detection etc., then ROI is used to extract the features and properties of the bone. Texture analysis is currently characterized the cancellous bone. Feature extraction methods are texture analysis which extract the features of bone and are classified as trabecular pattern indices, histomorphometric analysis and transform-based analysis. Finally, the bone is classified as healthy or osteoporotic and the performance can be defined interms of accuracy and precision. For example, an vertebrae of spine is considered which is shown in figure 4 and Spine ROI detection is shown in figure 5

First, the ROI of vertebrae is detected and it is a preprocessing step where image enhancement is done like removal of noise and other disturbances. In ROI detection, features of spine is extracted and enhanced. In this case, the foreground of image reduced using thresholding technique and further foreground will be enhanced by using histogram equalization method. The figure 6 demonstrates the segmentation results. A presegmentation method called as super pixel segmentation used to detect contour of each vertebra.

Figure 6: Comparison of Automated Segmentation (Right) with Manual segmentation (Left)



The Trabecular bone looks like structure of honey comb and it is more porous that is correlated to development of osteoporosis. The bone structure is characterized through different numerical methods called statistical methods and 255 texture analysis methods. Histogram features and statistical features allows evaluation of the texture of spongy bone. In pre processed stage, the square area extracted from distal radius to illustrate the texture of trabecular bone. To remove non-uniform illumination of the effects and to improve contrast between trabecular bone and surroundings a subtraction method was applied to the ROI using a Gaussian filter of SD as shown in figure 7.

Figure 7: Retrieval of distal radius ROI (Left) Identification of middle of circle and Retrieve the distal radius of ROI circle (Right)

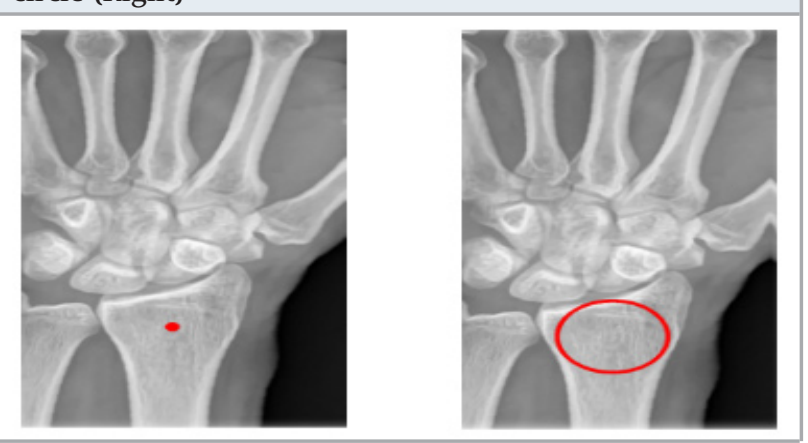


3. Literature Review: Bone density or BMD is measured using different methods or solutions for diagnosing osteopenia and osteoporosis. Osteopenia is a systematic disease that is defined by an overall loss of bone density which causes weak bones whereas Osteoporosis is not only the loss of bone but also becomes porous and likely to fracture which is shown in the figure 8. This two disease measured using different methods gives BMD that indicates the amount of mineral content present in the bone disease.

Figure 8: Osteopenia and Osteoporosis

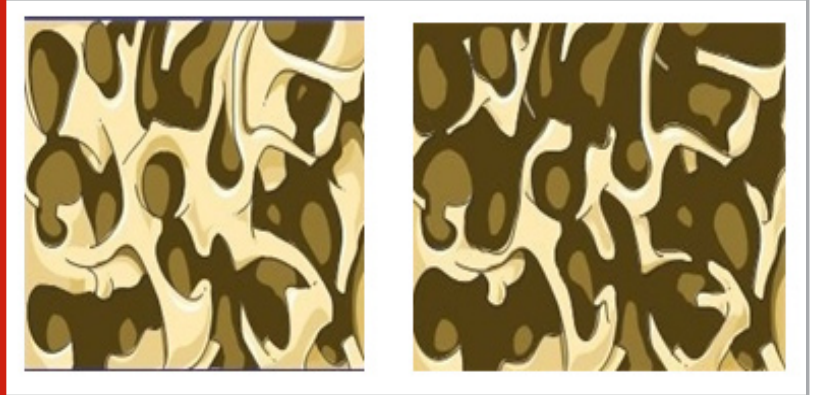

(Thamnawat et al. 2019) identified the bone density with different algorithms with low resolution of (ROI) of lumbar spine image obtained from the DEXA to predict the location of Vertebral bodies using different algorithms such as Bi-Histogram Equalization with Adaptive Sigmoid Functions, Density-Based and Ellipse and Object improvement techniques but these experimental results showed Area Overlap Ratio of 79.67\% and Precision value of 81.67\%. (Haba et al. 2019) diagnosed the bone density of human osteoarthritis femoral heads using a method called thermo gravimetric analysis (TGA). The proposed approach measures the BMD to show the evaluated correlation factor between DEXA, ashing and TGA. Using this correlation factor, very small bone samples were analyzed from a patient biopsy about BMD. In such cases, the human femoral head was considered and noted a significant loss in biological fraction from $200{ }^{\circ} \mathrm{C}$ to $600{ }^{\circ}$.

(Botha et al. 2011) was proposed a methodology to estimate age and gender of South African population using BMD values and linear regression analysis. Femur bone BMD values were obtained from DEXA.The Regression equations estimate the age from neck bone and the BMD values indicates a considerable variation between black and white groups, but the combined group these regression equations were less accurate. This would require more samples to differentiate a group. Braz (Liu et al. 2019) used a new technique to evaluate mechanical features of bone using acoustic radiation which leads to diagnosis of bone disease. Acoustic radiation force used for stimulating the medium and the nonlinear interactions within the bone tissue to produce lowfrequency signal (kHz). (Fathima et al. 2018) illustrated a methodology called $\mathrm{K}$-means clustering, mean-shift algorithm, and mathematical analysis called statistical analysis to assess BMD values. The BMD was detected through a DEXA scan which confirms the correct position of ROI of the patient. From Statistical analysis T-score and Z-the score was obtained.

(Harrar et al. 2018) uses logistic regression to evaluate various attributes of Osteoporosis such as texture of radiographic image and clinical parameters of two populations. To improve the ability to discriminate between groups, four models can be trained using logistic regression. With the help of the above technique, high performance can be achieved. Based on the above method $86.4 \%$ accuracy was obtained. To enhance rate of classification and need to construct more parameters and various classifiers are combined. (Areeckal and Kocher 2018) suggested different Segmentation methods like edge detection methods, region growing methods, etc., and similarly Texture analysis like Trabecular pattern indices, Histomorphometric method to predict osteoporosis of hip or spinal fractures. (Bhattacharya et al. 2019) was proposed automatic methodology for an early prediction of the radiographic bone image to determine whether patient is in osteoporotic or not, it is complex to note the X-rays in darkness instead of brightness.

A new approach is developed to detection bone fracture using various techniques like feature selection, classification, and machine learning classifiers such as support vector machine (SVM) and Nearest Neighbors(NN) were used. (Li et al. 2019) proposed a new approach which is a fully automated method Ultrasonic backscatter algorithms like-classical periogram for the measurement and estimation of cancellous bone, performance was improved. (Riaz et al. 2019) osteoporosis classification was carried out using combination of texture characteristics such as multi-resolution Gabor filters etc. This methodology demonstrate good classification with overall accuracy about $72.71 \%$ but because of lack of adequate data, accuracy has been reduced. Riad (Riad et al. 2019) characterize the cancellous bone from osteoporosis diagnosis using two well-known circular models. They are used to extract possible features and improve classification performance.

This approach identified the osteoporosis patients from both osteoporosis patients and control populations. (Vijay et al. 2019) proposed a method to identify BMD of ROI using DEXA of proximal femur namely, femur neck, Ward's triangle etc., but it cannot measure the volumetric BMD. Quantitative Computer Tomography (QCT) used measure cancellous BMD. The proximal CT image was analyzed using Hounsfield (HU) unit for upper ROI regions. The drawbacks observed are no people having previous osteoporotic fracture. (Braz et al. 2019) showed the Modified Gabor filter and Ellipse analysis to estimate the Vertebrae Pose but this method cannot work with high-level damage of vertebrae pose from lesion and bone collapse. Chengcheng (Liu et al. 2019) proposed a methodology to diagnose microstructures of cancellous bone using ultrasonic backscatter. With the help of 2.25 $\mathrm{MHz}$ transducer in addition to ultrasonic backscatter 33 bovine cancellous bone measurement can be performed. The results demonstrated would provide some additional 
information of structural bone and bone density features that were not related to the BMD measurement.

(Saenpaen 2018) compared image enhancement techniques like Brightness Preserving Dynamic Fuzzy Histogram Equalization Histogram Equalization(HE) etc., by considering the lumbar spine X-ray image. (Juliastuti, et al. 2013) suggested algorithms for early detection of Osteoporosis of Mandibular bone which improves the ability of the system but the bone mineral density using Gray Level Co-occurrence Matrix(GLCM) and Support Vector Machine (SVM) but it doesn't give 100 $\%$ results which are due movement of the patient head and therefore output could get poor image quality. (Khan et al. 2017) proposed an image processing algorithm for estimating BMD using digital X-ray images but Graphic User Interface was needed. Fong Choong (Fong et al. 2018) illustrated the statistical analysis of low BMD of epileptic children (neurological disorder) with long-term Antiepileptic Drugs (AED) and this can be diagnosed using DEXA that measures child lumbar spine.

This implemented by considering two factors one is extrinsic (environmental factor) and the other is intrinsic (genetic factor). Ohashi (Ohashi et al. 2018)investigated the osteopenia of spinal fusion with adolescent idiopathic scoliosis AIS (is an abnormal curvature of spine) using statistical analysis. The pre and post-surgical values were estimated using Wilcoxon signed rank test. The limitations of this paper are no BMD data is available during the surgery and therefore BMD has not been evaluated. (Tejaswini et al. 2016) proposed ANN, which is a decision-making system to detect and predict osteoporosis using LabVIEW.

This method is found to be expensive and requires experts to deal with the types of equipment. Coupled (Coupaud et al. 2015) demonstrated peripheral Quantitative Computed Tomography (pQCT) to diagnose spinal cord lesion that is an unused type of osteoporosis that affects the paralyzed limbs. (Dendere et al. 2015) illustrated a method that acquired dual energy images of middle finger using two detectors of slot scanning radiography system. The advantage of this method is that it doesn't require pulse counting detectors that are necessary in K-edge filtering. But these detectors were determined that whether the photon is considered to be high or low-energy photon. The drawback of this method is that it has less spectral separation than the K-edge filtering technique.

4. Inferences of DXA Or DEXA Over Normal X-Ray Machines: Evaluation of BMD with DEXA doesn't provide accurate fracture risk people with progressive changes due to following reasons like corticosteroids (reduce immune system) and a small or large body weight. The corticosteroids and progressive changes bones become denser. Therefore, DEXA evaluate the possibilities of fractures. On the other hand, the risk of fracture is estimated by DEXA in persons with small bones. Compared to DEXA there are several advantages of normal X-ray machines:

1. Portable and take less than 20 minutes for detecting bone fracture.

2. There is a reduction in radiation exposure.

3. The front panel of portable X-ray machine protects against scattered radiation.

4. For Bone Density test - Effective Radiation Dose is $0.001 \mathrm{mSV}$ but in the case of DXA it may vary from $0.013 \mathrm{mSv}$ to $0.3 \mathrm{mSv}$

\section{CONCLUSION}

Inferences from reviewed papers, the authors summarize about diagnosing of osteoporosis using DEXA scan which helps to prevent early facture of bone and to inform the patients about these risk factor based on consideration of T-score and Z-score. This literature considered different methods and solutions like Bi-Histogram Equalization with adaptive sigmoid, density-based ellipse techniques, linear regression analysis, logistic regression, segmentation methods, and texture analysis for diagnosis of osteoporosis. But it requires a lot of data and unable to detect a high level of damage of bone and lesion and in literature average accuracy was noted between 75 $\%$ to $85 \%$.To get adequate accuracy and precision, the methodologies like preprocessing (Low Pass Filter and High Pass Filter), Color Image Analysis, Particle Swarm Optimization, Efficient LBP and Pixel Wise LBP, etc., may propose for diagnosing osteoporosis.

\section{REFERENCES}

Areeckal, A.S. and Kocher, M.,Current and emerging diagnostic imaging-based techniques for assessment of osteoporosis and fracture risk. IEEE reviews in biomedical engineering, 12, pp.254-268,(2018).

Bhattacharya, S., Nair, D., Bhan, A. and Goyal, A., Computer Based Automatic Detection and Classification of Osteoporosis in Bone Radiographs. In 2019 6th International Conference on Signal Processing and Integrated Networks (SPIN) IEEEE,pp. 1047-1052, ( 2019) Bone mineral density after spinal fusion surgery for adolescent idiopathic scoliosis at a minimum 20-year follow-up. Spine deformity, 6(2), (pp.170-176),2018.

Botha, D., Lynnerup, N. and Steyn, M.,. Age estimation using bone mineral density in South Africans. Forensic science international, 297, pp.307-314,(2019).

Braz, G.A., Agnollitto, P.M., Nogueira-Barbosa, M.H., Pavan, T.Z. and Carneiro, A.A., 2019, October. Diagnosis of osteoporosis using the low frequency acoustic response of mice femoral bones irradiated by a high frequency acoustic radiation pulse.IEEE International Ultrasonics Symposium (IUS) , pp. 529-531,(2019) Coupaud, S., McLean, A.N., Purcell, M., Fraser, M.H. and Allan, D.B.,Decreases in bone mineral density at cortical and trabecular sites in the tibia and femur during the first year of spinal cord injury. Bone, 74, (pp.69-75), 2015

Dendere, R., Potgieter, J.H., Steiner, S., Whiley, S.P. and Douglas, T.S.,Dual-energy x-ray absorptiometry for measurement of phalangeal bone mineral density on a slot-scanning digital radiography system. 
IEEE Transactions on Biomedical Engineering, 62(12),(pp.2850-2859), 2015.

Fathima, S.N., Selvi, R.T. and Beham, M.P., Assessment of BMD and Statistical Analysis for Osteoporosis Detection. Biomedical and Pharmacology Journal, 12(4), pp.1907-1914, ( 2019.)

Fong, C.Y., Kong, A.N., Noordin, M., Poh, B.K., Ong, L.C. and Ng, C.C.,Determinants of low bone mineral density in children with epilepsy. European Journal of Paediatric Neurology, 22(1), (pp.155-163),2018.

Haba, Y., Köckerling, M., Schick, C. and Bader, R., 2019, May. Determination of Bone Density and Bone Composition Using Thermoaravimetric Analysis. 12th International Conference on Measurement. IEEE pp. 178-181 (2019)

Harrar, K., Jennane, R., Zaouchi, K. and Lespessailles, E.,Discrimination of Osteopathic Patients using Logistic Regression Model. International Conference on Signal, Image, Vision and their Applications (SIVA) IEEE,pp. 1-5,(2018).

Juliastuti, E., Diputra, Y. and Mayantasari, M.,Dental panoramic image analysis onmandibular bone for osteoporosis early detection.3rd International Conference on Instrumentation Control and Automation (ICA),IEEE,(pp. 138-143),2013

Khan, S.S., Jayan, A.S. and Nageswaran, S.,An image processing algorithm to estimate bone mineral density using digital X-ray images. Second International Conference on Electrical, Computer and Communication Technologies (ICECCT),IEEE, (pp. 1-4),2017.

Li, B., Xu, F., Liu, C., Li, D., Le, L.H., Ta, D. and Wang, W.,Effect of spectral estimation on ultrasonic backscatter parameters in measurements of cancellous bones. IEEE Access, 7, pp.83034-83045,(2019).

Liu, C., Li, B., Diwu, Q., Li, Y., Zhang, R., Ta, D. and Wang, W., Relationships of ultrasonic backscatter with bone densities and microstructure in bovine cancellous bone. IEEE Transactions on Ultrasonics, Ferroelectrics, and Frequency Control, 65(12),(pp.2311-2321),2018.

Ohashi, M., Hirano, T., Watanabe, K., Katsumi, K., Shoji, H., Mizouchi, T. and Endo, N.,

Parameshachari, B.D., Panduranga, H.T. and liberata Ullo, S., 2020, September. Analysis and Computation of Encryption Technique to Enhance Security of Medical Images. In IOP Conference Series: Materials Science and Engineering (Vol. 925, No. 1, p. 012028). IOP Publishing.

Prabu, S., Balamurugan, V. and Vengatesan, K., 2019. Design of cognitive image filters for suppression of noise level in medical images. Measurement, 141, pp.296301.

Riad, R., Jennane, R., Douzi, H., Rafiki, A., Lespessailles, E., Bruno, O.M. and El Hassouni, M.,A New Complex Wavelet Relative Phase for Osteoporosis Diagnosis. 4th World Conference on Complex Systems (WCCS),IEEE,pp. 1-6,2019

Riaz, F., Nemati, R., Ajmal, H., Hassan, A., Edifor, E. and Nawaz, R.,. Osteoporosis Classification Using Texture Features. In 2019 IEEE 32nd International Symposium on Computer-Based Medical Systems (CBMS), IEEE,pp. 575-579,(2019)

Saenpaen, J., Arwatchananukul, S. and Aunsri, N.,A Comparison of Image Enhancement Methods for Lumbar Spine X-ray Image.15th International Conference on Electrical Engineering/Electronics, Computer, Telecommunications and Information Technology (ECTI-CON),IEEE, (pp. 798-801),2018

Tejaswini, E., Vaishnavi, P. and Sunitha, R.,Detection and prediction of osteoporosis using impulse response technique and artificial neural network.International Conference on Advances in Computing, Communications and Informatics (ICACCI), IEEE. (pp. 1571-1575),2016. Thamnawat, S., Ham, J.G. and Rasmequan, S., 2019, October. Region of Interest Identification on LowResolution Lateral Spine Radiography Image using Density-based and Ellipse-like Method. 4th International Conference on Information Technology (InCIT) IEEE,pp. 77-82,(2019)

Vijay, A., Shankar, N., Ligesh, C.A.S. and Anburajan, M.,Evaluation of osteoporosis using CT image of proximal femur compared with dual energy X-ray absorptiometry (DXA) as the standard.3rd International Conference on Electronics Computer Technology,IEEE (Vol. 3, pp. 334-338),2011

Yookwan, W., Chinnasarn, K. and Jantarakongkul, B., 2018, Automated Vertebrae Pose Estimation in Low-Radiation Image using Modified Gabor Filter and Ellipse Analysis5th International Conference on Advanced Informatics: Concept Theory and Applications (ICAICTA),IEEE,(pp. 141-146),2018 